\title{
Computational study of the ro-vibrational spectrum of $\mathrm{CO}-\mathrm{CO}_{2}$
}

\author{
Eduardo Castro-Juárez, Xiao-Gang Wang, and Tucker Carrington Jr.* \\ Chemistry Department, Queen's University, Kingston, Ontario K7L 3N6, Canada
}

Ernesto Quintas-Sánchez and Richard Dawes
Department of Chemistry, Missouri University of Science and Technology, Rolla, Missouri 65409

(Dated: August 3, 2019)

\begin{abstract}
An accurate $a b$ initio ground-state intermolecular potential energy surface (PES) was determined for the $\mathrm{CO}-\mathrm{CO}_{2}$ Van der Waals dimer. The Lanczos algorithm was used to compute ro-vibrational energies on this PES. For both the $\mathrm{C}$-in and the $\mathrm{O}$-in T-shaped isomers, the fundamental transition frequencies agree well with previous experimental results. We confirm that the in-plane states previously observed are geared states. In addition, we have computed and assigned many other vibrational states. The rotational constants we determine from $J=1$ energy levels agree well with their experimental counterparts. Planar and out-ofplane cuts of some of the wavefunctions we compute are quite different, indicating strong coupling between the bend and torsional modes. Because the stable isomers are T-shaped, vibration along the out-of-plane coordinates is very floppy. In CO- $\mathrm{CO}_{2}$, when the molecule is out-of-plane, interconversion of the isomers is possible, but the barrier height is higher than the in-plane geared barrier height.
\end{abstract}

\footnotetext{
*Electronic address: Tucker. Carrington @queensu . ca
} 


\section{INTRODUCTION}

$\mathrm{CO}-\mathrm{CO}_{2}$ is a Van der Waals dimer composed of the two monomers $\mathrm{CO}$ and $\mathrm{CO}_{2}$, both of which are of astrophysical interest. Its infra-red and microwave spectra have been recorded and its structure determined.[1-3] $\mathrm{CO}-\mathrm{CO}_{2}$ has two stable isomers. Both are T-shaped with the $\mathrm{CO}_{2}$ monomer at the top of the $\mathrm{T}$ and the $\mathrm{CO}$ monomer the stem of the $\mathrm{T}$. The lower-energy isomer has the $\mathrm{C}$ of $\mathrm{CO}$ close to the $\mathrm{C}$ of $\mathrm{CO}_{2}$. The higher-energy isomer has the $\mathrm{O}$ of $\mathrm{CO}$ close to the $\mathrm{C}$ of $\mathrm{CO}_{2}$. We shall refer to these isomers as $\mathrm{C}$-in and $\mathrm{O}$-in. Both isomers are shown on the right side of Figure 1. The C-in isomer was first studied by Legon and Suckley[1] and later by others. [4, 5] The existence of the O-in isomer was predicted by ab initio calculations [6] and later confirmed.[2] For both isomers, two inter-monomer transition frequencies have been observed. One is for an in-plane state and the other for an out-of-plane state.[2, 3] Harmonic frequencies have also been computed with ab initio methods.[5-7] Although the experimental frequencies for the lower energy $\mathrm{C}$-in isomer are rather close to the $a b$ initio harmonic frequencies, the experimental $\mathrm{O}$-in frequencies are not close to the harmonic values. In the $\mathrm{C}$-in case, the agreement is good enough that the experimental in-plane vibration was assigned to the "CO rock/geared bend". Van der Waals molecules have been avidly studied by experimentalists and theorists for decades. [8$10]$

In this paper, we report a new four-dimensional (4-D) ab initio potential energy surface (PES) that is a function of the intermolecular coordinates of $\mathrm{CO}-\mathrm{CO}_{2}$, and energy levels computed on it. The PES is built using points computed at the CCSD(T)-F12b/VTZ-F12 level. The only approximation in the energy-level calculation is the separation of the high frequency intra-monomer coordinates from the low frequency inter-monomer coordinates. Energy levels and wavefunctions are computed with the Lanczos method and a large spherical-harmonic type basis. It has been demonstrated that such calculations are accurate for other Van der Waals dimers. [11-14]

Probability Density (PD) and wavefunction cut plots are used to label the energy levels. We find significant coupling between in-plane and out-of-plane coordinates. The experimental transition frequencies are actually from combination bands, but they are expected to be very close to the fundamentals we calculate. 


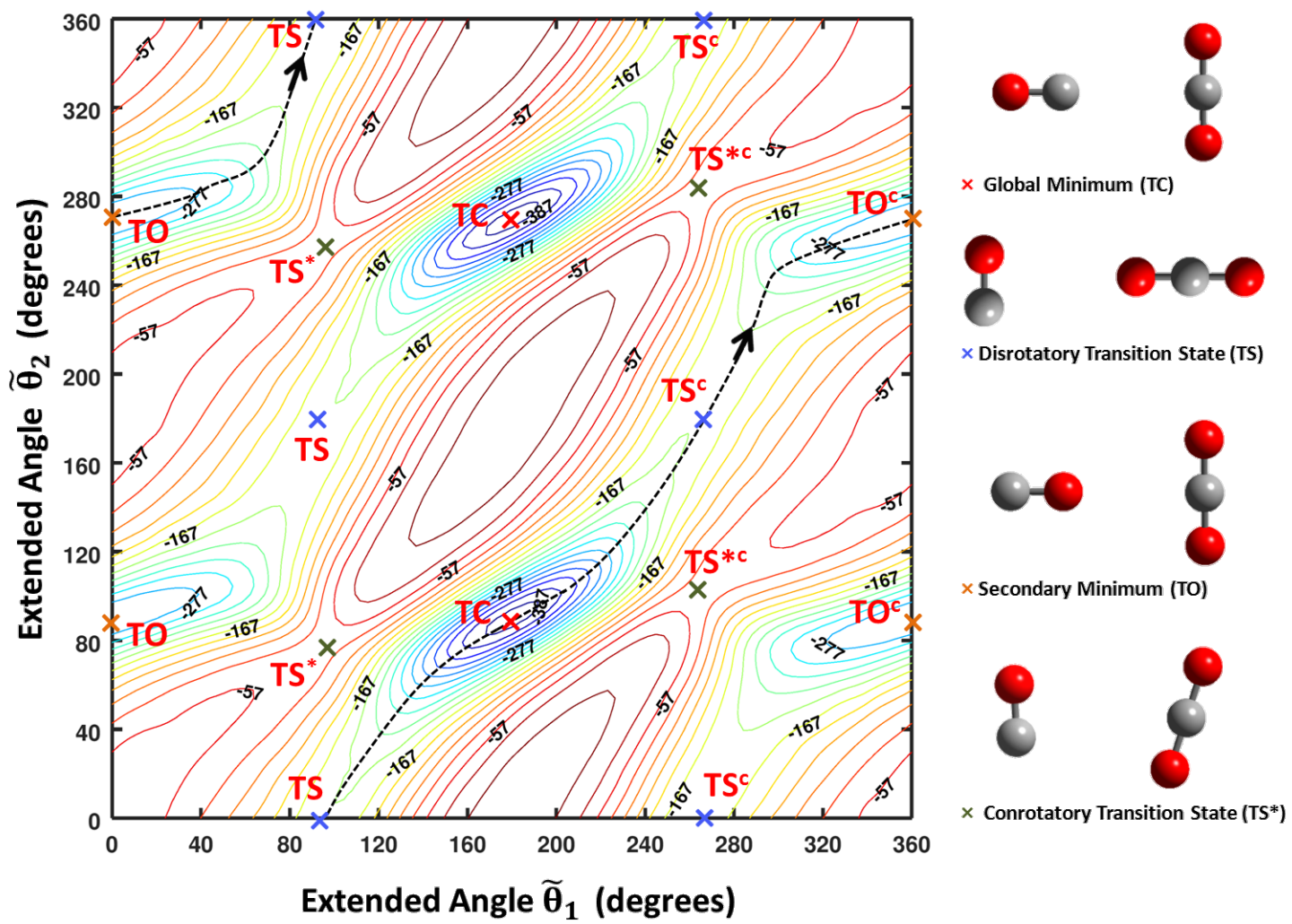

FIG. 1: $r_{0}$-optimized contour plot of the PES as a function of the extended angles $\tilde{\theta}_{1}$ and $\tilde{\theta}_{2}$. For each pair of angles, the energy (given in $\mathrm{cm}^{-1}$ ) is optimized with respect to the center-of-mass distance $r_{0}$. The position of stationary points—and the corresponding molecular configuration—is highlighted. The extended-angle coordinates are similar to those described elsewhere.[15] Every structure with a $c$ superscript, on the right side, is a copy of a structure on the left side. TS and $\mathrm{TS}^{c}$ label geared transition states; $\mathrm{TS}^{*}$ and $\mathrm{TS}^{* c}$ are the anti-geared transition states. The dashed curve shows the geared path for the molecule, from TC, to TS ${ }^{c}$, to $\mathrm{TO}^{c}$, to TS and back to TC.

\section{IMLS PES FITTING}

The coordinates used to define the 4-D intermolecular $\mathrm{CO}-\mathrm{CO}_{2}$ potential: $r_{0}, \theta_{1}, \theta_{2}$, and $\phi_{2}$, are depicted in Figure 2. $\vec{r}_{0}$ is the vector from the centre of mass of CO to the centre of mass of $\mathrm{CO}_{2}, \vec{r}_{1}$ and $\vec{r}_{2}$ are vectors aligned with the monomers. $r_{0}$ is the length of $\vec{r}_{0}$, and $\theta_{1}$ and $\theta_{2}$ are (respectively) the angles between $\vec{r}_{0}$ and the vectors $\vec{r}_{1}$ and $\vec{r}_{2}$. The fourth coordinate is the dihedral (out of plane) torsional angle, labeled $\phi_{2}$, which is the angle between the vectors $\vec{r}_{0} \times \vec{r}_{1}$ and $\vec{r}_{0} \times \vec{r}_{2}$ 


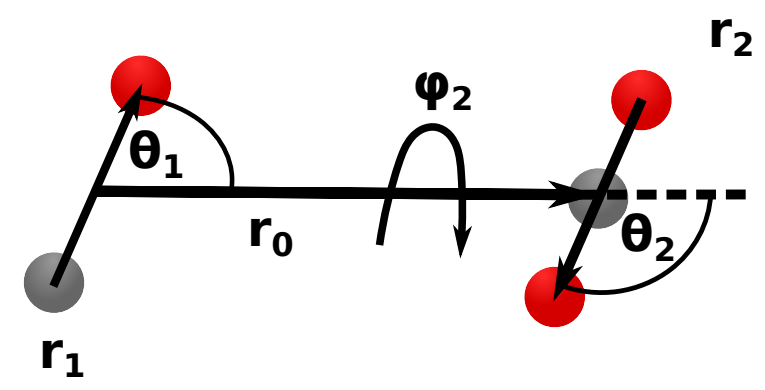

FIG. 2: Coordinates used to describe $\mathrm{CO}-\mathrm{CO}_{2} . r_{0}$ : center-of-mass separation (length of $\overrightarrow{r_{0}}$ ); $\theta_{1}$ and $\theta_{2}$ : angles between $\vec{r}_{0}$ and the vectors $\vec{r}_{1}$ and $\vec{r}_{2}$ respectively; and $\phi_{2}$ : torsional angle.

Both monomers were held rigid. As in previous studies, $[15,16]$ for the $\mathrm{CO}$ molecule we used $r_{1}=1.1282 \AA$, corresponding to the rotational constant $B=1.9317 \mathrm{~cm}^{-1}$. $[16,17] \mathrm{CO}_{2}$ is linear, with each CO bond-distance fixed at $r_{C O}=1.162086 \AA$, which is consistent with the experimental rotational constant $0.39021894 \mathrm{~cm}^{-1}$. $[18,19]$ Masses of $15.9949146221 \mathrm{u}$ and $12 \mathrm{u}$ were used for ${ }^{16} \mathrm{O}$ and ${ }^{12} \mathrm{C}$, respectively. All ab initio calculations were performed using the Molpro electronic structure code package.[20] A lower level guide surface was constructed using data at the explicitly correlated CCSD(T)-F12a/VDZ-F12 level.[21] Data for the final high-level PES was generated using the CCSD(T)-F12b/VTZ-F12 method and basis set.[22]

The 4-D PES was constructed using an automated interpolating moving least squares method, which has been used in several previous studies [15, 23-25] and has been recently released as a software package under the name AUTOSURF.[26, 27] This interpolative approach can accommodate arbitrary energy-surface topographies and is particularly advantageous in cases with large anisotropy, which are challenging for traditional Legendre expansions. The shortest intermonomer center-of-mass distance considered is $R=2.1 \AA$, with the additional restriction of a maximum repulsive energy of $6 \mathrm{kcal} / \mathrm{mol}\left(\sim 2100 \mathrm{~cm}^{-1}\right)$ above the separated monomers asymptote. To guide the placement of high-level data - and avoid computing and discarding computationally expensive $a b$ initio energies in highly repulsive regions - an initial lower-level guide surface was constructed using a set of 1949 symmetry-unique points, distributed using a Sobol sequence biased to sample the short range region more densely. For the high-level PES, the global estimated root-meansquared fitting error tolerance was set to $0.2 \mathrm{~cm}^{-1}$ and the total number of automatically generated symmetry-unique points needed to reach that target was 2654 . The ab initio data coverage of the fitted PES extends to $R=20.0 \AA$. A local fit was expanded about each data point, and for each of the local fits, a fitting basis of 301 functions was used. The final potential is obtained as the 
normalized weighted sum of the local fits.

Figure 1 shows the PES in planar configurations. The plot is made by finding the value of $r_{0}$ that minimizes the energy for each $\theta_{1}, \theta_{2}$ pair. The plot is in the extended coordinates, $\left(\tilde{\theta}_{1}, \tilde{\theta}_{2}\right)$. We use extended coordinates similar to those defined in Ref. 15. They are not identical because we want the range of $\left(\tilde{\theta}_{1}, \tilde{\theta}_{2}\right)$ to be from 0 to $2 \pi$ so that wavefunctions on extended plots appear in the middle, and not at the edges. The extended coordinates are also used to make wavefunction plots. They are defined in Figure 3.

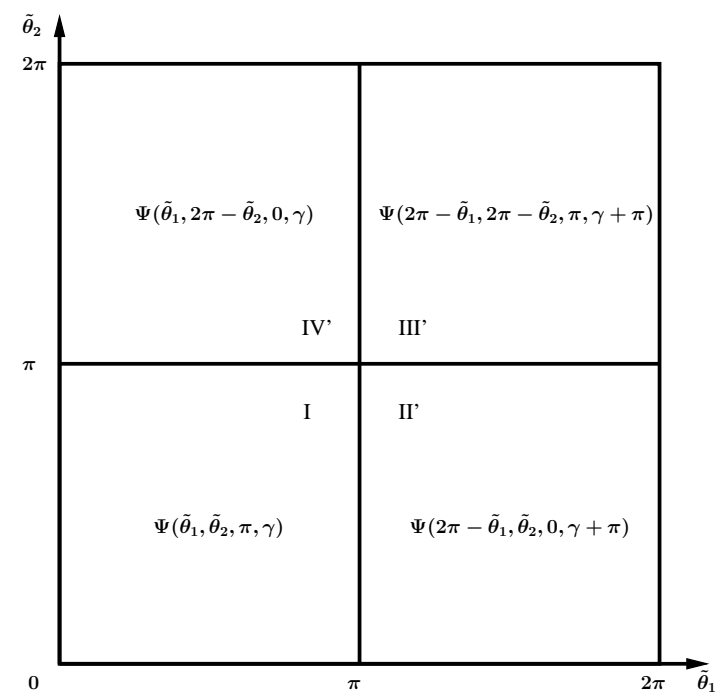

FIG. 3: Definition of the extended angles $\left(\tilde{\theta}_{1}, \tilde{\theta}_{2}\right)$ in the $[0,2 \pi]$ range. In our convention, monomer 1 is left of monomer 2 and the positive directions for $\tilde{\theta}_{1}$ and $\tilde{\theta}_{2}$ are clockwise and counter-clockwise, respectively. Given in each quadrant is the computed wavefunctions $\Psi\left(\theta_{1}, \theta_{2}, \phi_{2}, \gamma\right)$. By equating the first (second) argument in $\Psi$ in the figure with $\theta_{1}\left(\theta_{2}\right)$ one obtains the definition of the extended coordinates in each quadrant. For example, in the bottom right quadrant, $\tilde{\theta}_{1}=2 \pi-\theta_{1}$. The definitions here complement the definitions of the extended angles in the $[-\pi, \pi]$ range given Ref. 15. Quadrants II', III', IV' are obtained from quadrants II, III, IV of Ref. 15 by shifting one or two angles by $2 \pi$.

The advantage of using extended coordinates is that it is easy to visualize a planar motion during which $\phi_{2}$ changes from $0^{\circ}$ to $180^{\circ}$. For example, $\mathrm{TC}$ (the global minimum), $\mathrm{TS}^{c}, \mathrm{TO}^{c}$, $\mathrm{TO}$, and TS are points on a disrotatory (or geared) cycle. The corresponding geared coordinate is $Q_{g}=\theta_{1}+\theta_{2}$. The path is easily identified in extended coordinates, but one must exit the plot on the right at $\mathrm{TO}^{c}$ and re-enter on the left at TO and also exit at TS at the top of the plot and re-enter at TS at the bottom of the plot. If extended coordinates were not used then to trace this path one would need 
TABLE I: Equilibrium and transition state geometries and energies for configurations shown in Figure 1 Absolute energies are relative to the asymptote. $\theta_{1}, \theta_{2}$, and $\phi_{2}$ are in degrees and $r_{0}$ is in Angström. $\phi_{2}$ for TC and TO is undefined.

\begin{tabular}{|c|c|c|c|c|}
\hline Stat. & $\left(\theta_{1}, \theta_{2}, \phi_{2}, r_{0}\right)$ & Ab initio $\left(\theta_{1}, \theta_{2}, \phi_{2}, r_{0}\right)[6]$ & $E_{a b s}\left(\mathrm{~cm}^{-1}\right)$ & $E_{\text {rel }}\left(\mathrm{cm}^{-1}\right)$ \\
\hline $\mathrm{TC}$ & $(180,90,-, 3.868)$ & $(180,90,-, 3.957)$ & -398.3 & 0 \\
\hline $\mathrm{TO}$ & $(0,90,-, 3.534)$ & $(0,90,-, 3.610)$ & -297.8 & 100.43 \\
\hline $\mathrm{TS}$ & $(90,180,0,4.434)$ & & -180.0 & 218.3 \\
\hline$X$ & $(90,90,90,3.529)$ & & -150.1 & 248.2 \\
\hline $\mathrm{TS}^{*}$ & $(92,76,0,6.951)$ & & -97.5 & 300.8 \\
\hline
\end{tabular}

to exit and re-enter at the middle of the plot. A disadvantage of using extended coordinates is that every point on the actual PES appears twice in Figure 1. Not counting copies, there are two minima: labelled "TC" (T-shaped C-in) and "TO" (T-shaped O-in) in the plot; and two saddle points: labelled TS* and TS. Note that the top half of Figure 1 is equivalent to the bottom half because of the symmetry of $\mathrm{CO}_{2}$. The minima of the $\mathrm{C}$-in and $\mathrm{O}$-in wells are at $-398.273 \mathrm{~cm}^{-1}$ and $-297.843 \mathrm{~cm}^{-1}$ respectively, both with respect to the dissociation energy of the complex. Geared and anti-geared cycles are prominent on the PESs of many dimers.[24, 25, 28, 29] However, the cycle is different in the $\mathrm{CO}-\mathrm{CO}_{2}$ case because the minima are not slipped parallel but T-shaped. The geometries and energies relative to the dissociation energy of the minima are given in Table I. Venayagamoorthy and Ford report that the energy difference between the two minima is 99.65 $\mathrm{cm}^{-1}$, whereas our difference is $100.43 \mathrm{~cm}^{-1}$. The TS saddle point is $218.3 \mathrm{~cm}^{-1}$ higher than TC. The states we can label (vide infra) are all localized in either the O-in well or the C-in well. Because the TS* saddle point is $300.8 \mathrm{~cm}^{-1}$ higher than TC, the anti-geared path has no influence on the low-lying levels.

There is also an out-of-plane path between TC and TO. It is evident in Figure 4 a, which shows the PES as a function of $\theta_{1}$ and $\phi_{2}$, with $\theta_{2}$ fixed at its value at the bottom of the C-in well and $r_{0}$ minimized, but restricted to be between the equilibrium values for both isomers. There is clearly a low-lying path along $\phi_{2}=90^{\circ}$ from $\theta_{1}=\varepsilon$ to $\theta_{1}=180^{\circ}-\varepsilon$, where $\varepsilon$ is some small value. At $\theta_{1}=180^{\circ}$ and $\theta_{1}=0^{\circ}, \phi_{2}$ is undefined. As $\theta_{1}$ approaches $180^{\circ}$ and $0^{\circ}$, the PES becomes independent of $\phi_{2}$ and it is therefore easy to slip into an out-of-plane configuration from a Tshaped geometry. The saddle point of this path is $248.2 \mathrm{~cm}^{-1}$ above TC. The same path is evident 

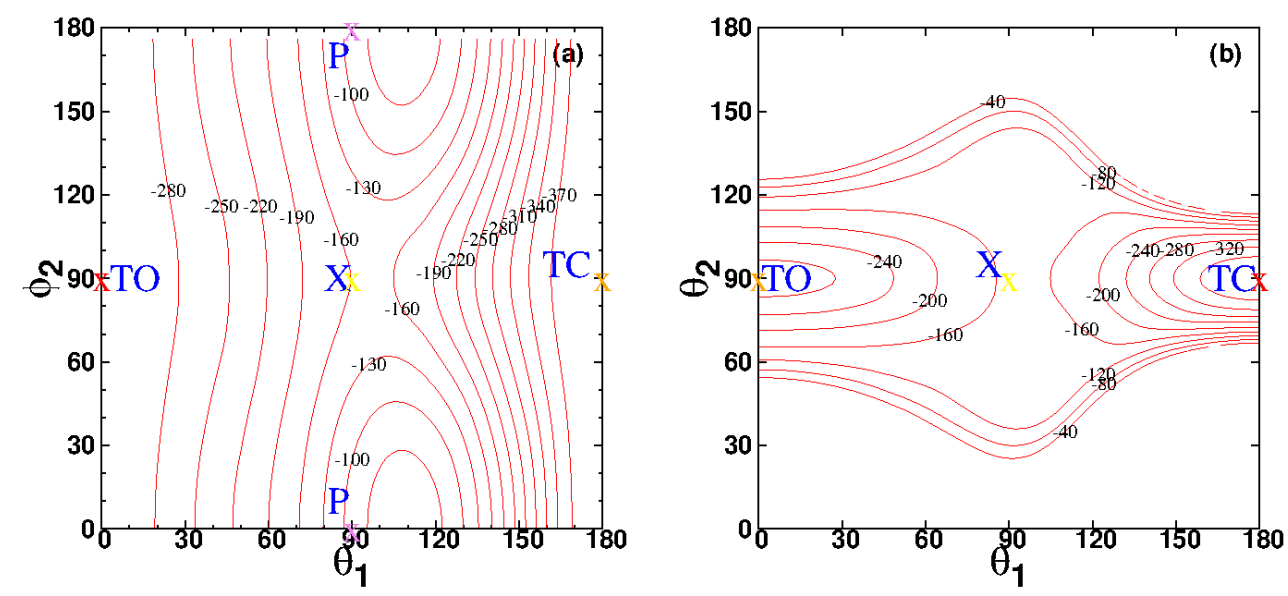

FIG. 4: Two plots showing the out-of-plane path connecting the two isomers. a) PES as a function of $\theta_{1}$ and $\phi_{2}$, with $\theta_{2}=90^{\circ}$, and $r_{0}$ equal to the value that minimises the energy, but with the restriction that $r_{0}$ must be between the equilibrium values for the isomers. $\mathrm{X}$ labels a shape where both isomers form a "cross" shape, and P labels a configuration in which both isomers are in the same plane and parallel to each other with $\theta_{1}=\theta_{2}=90^{\circ}$. Due to the symmetry of $\mathrm{CO}_{2}$, the value of the potential is identical at $\phi_{2}$ and $360^{\circ}-\phi_{2}$ and only the symmetrically unique part of the PES is shown. $\phi_{2}$ is undefined at TC and TO, but when $\theta_{1}=180^{\circ}-\varepsilon$, or $\varepsilon$ where $\varepsilon$ is some small angle, the shape of the molecule is almost the same for all $\phi_{2}$ values and TO and TC labels could therefore be put anywhere along the $\phi_{2}$ axis. We put the labels near the ends of the path at about $\phi_{2}=90^{\circ}$. b) PES as a function of $\theta_{1}$ and $\theta_{2}$, with $\phi_{2}=90^{\circ}$, and $r_{0}$ equal to the value that minimises the energy, with the restriction that $r_{0}$ must be between the equilibrium values for both isomers.

in Figure $4 \mathrm{~b}$, which shows the PES as a function of $\theta_{1}$ and $\theta_{2}$ with $\phi_{2}$ fixed at 90 and $r_{0}$ minimized, but restricted to be between the equilibrium values for both isomers. As that figure shows, when $\phi_{2}=90^{\circ}$ there is little coupling between $\theta_{1}$ and $\theta_{2}$. When $\phi_{2}=0^{\circ}, \theta_{1}$ and $\theta_{2}$ are strongly coupled and the natural motion is along the geared and anti-geared $\left(Q_{a}=\theta_{1}-\theta_{2}\right)$ coordinates (see Figure 1). When the two monomers are in the same plane, $\theta_{1}$ and $\theta_{2}$ are coupled because the monomers push against each other. When the two monomers are not in the same plane, $\theta_{1}$ and $\theta_{2}$ are not coupled because the monomers do not obstruct each other. Such an out-of-plane path between T-shaped minima might also be important for other Van der Waals molecules. In $\mathrm{CO}-\mathrm{CO}_{2}$, the fact that $\phi_{2}$ is very floppy manifests itself in the wavefunctions, see Figure 5.

Figure 6 shows the potential as a function of $r_{0}$ upon approach toward each of the stationary 

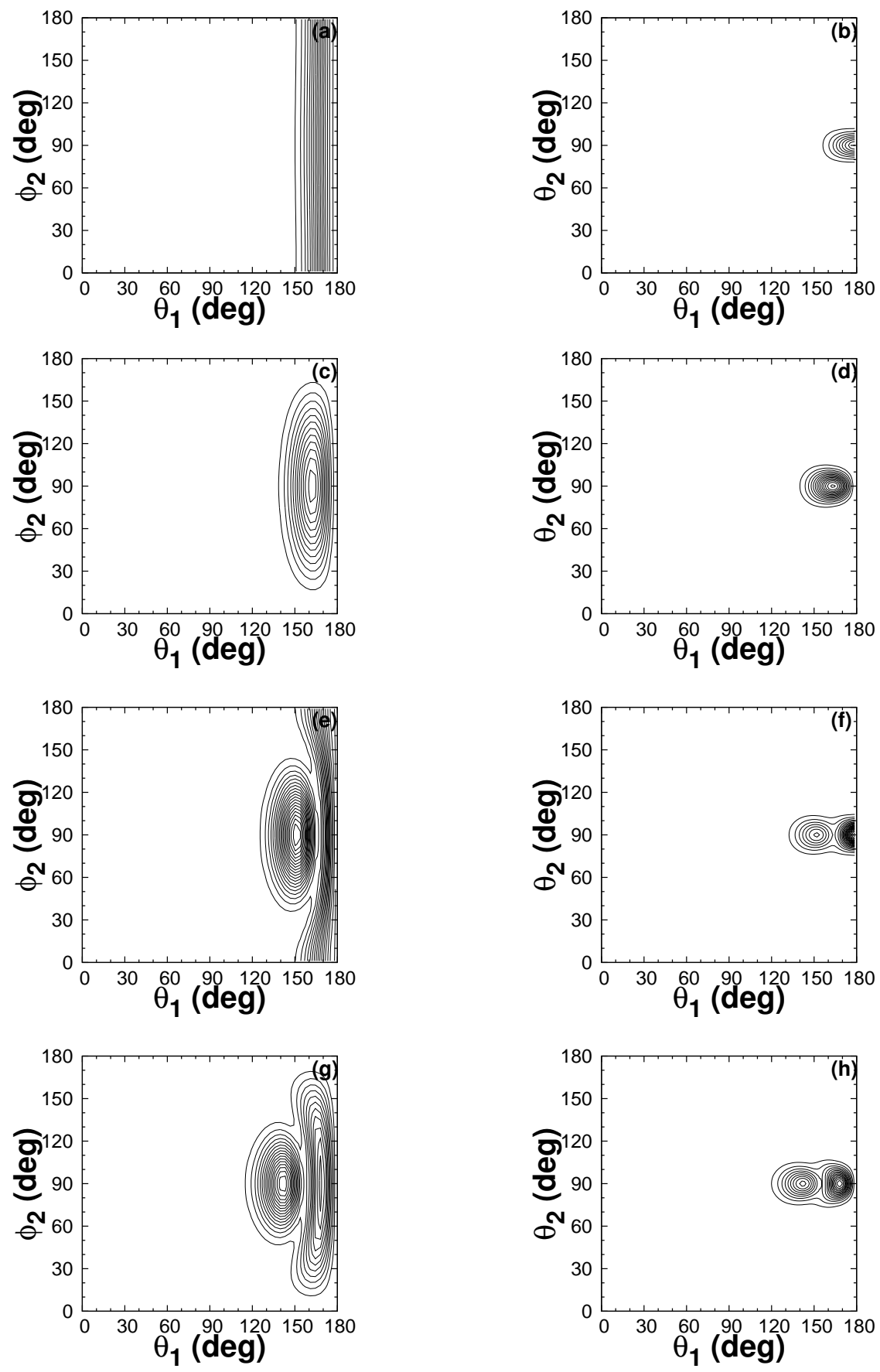

FIG. 5: Probability density plots for the ground state and the first three out-of-plane states (1B-, 5A+ and 6B-) whose energies are $0.0000,44.5664,86.0042$ and $123.3091 \mathrm{~cm}^{-1}$, from top to bottom. The left column shows PD plots that are functions of $\theta_{1}$ and $\phi_{2}$, whereas the right column shows PD plots that are functions of $\theta_{1}$ and $\theta_{2}$. Due to the symmetry of $\mathrm{CO}_{2}$, the value of the potential is identical at $\phi_{2}$ and $360^{\circ}-\phi_{2}$ and only the symmetrically unique part of the PES is shown. 


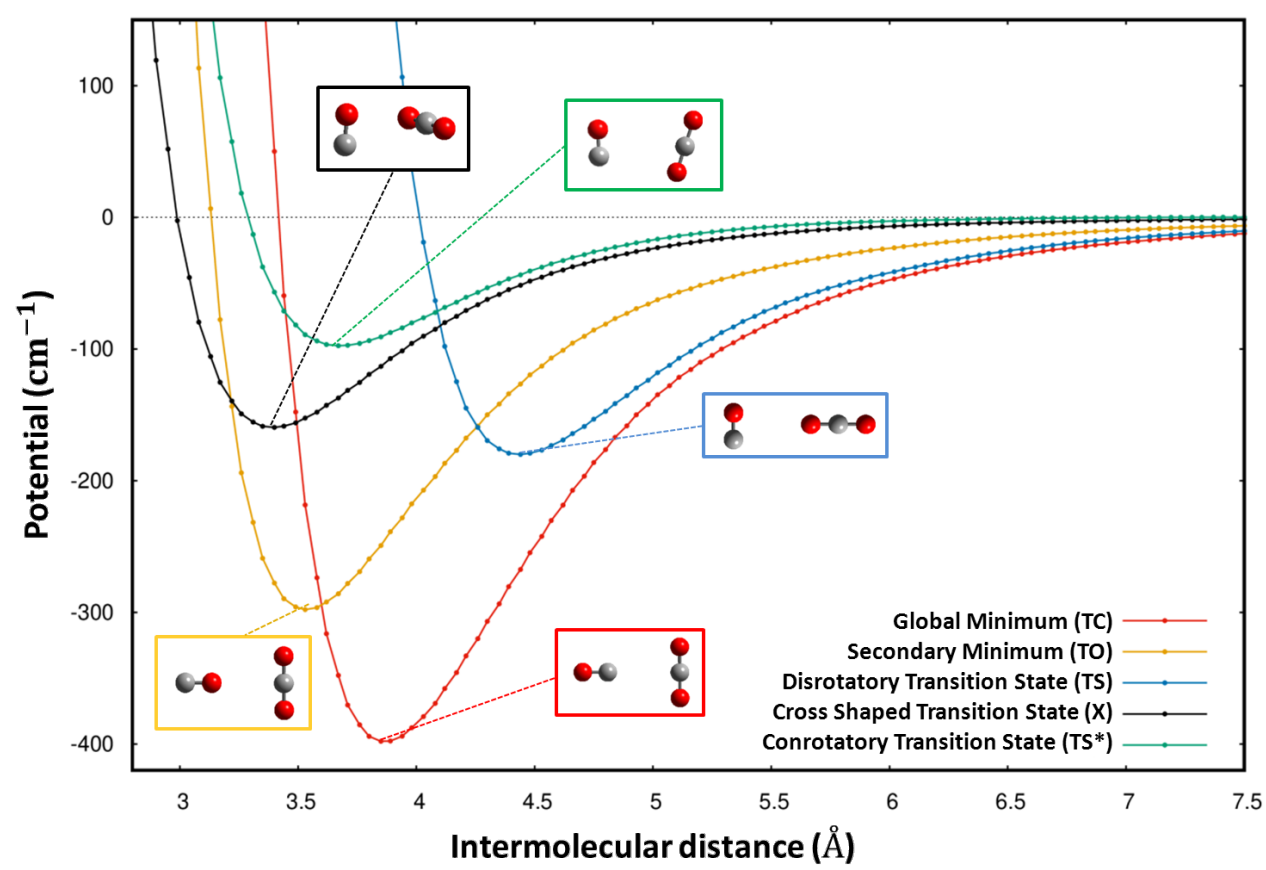

FIG. 6: Radial cuts through the PES for the stationary points labelled in Figure 1

points highlighted in Figure 1. The variation in those cuts gives some indication of the anisotropy of the interactions. To ensure correct symmetry properties, the representation of the PES was symmetrized to provide numerically exact permutation symmetry with respect to exchange of the two $\mathrm{O}$-atoms in $\mathrm{CO}_{2}$. On the quadrature grid the largest difference between potential values that should be identical is $0.0076904 \mathrm{~cm}^{-1}$.

\section{SOLVING THE VIBRATIONAL SCHRÖDINGER EQUATION}

We solve the Schrödinger equation retaining only the four inter-monomer coordinates by using a ro-vibrational basis and the Lanczos method. [28, 30-37] The kinetic energy operator in the Jacobi coordinates of Figure 2 is well known.[35, 38, 39] For the rotational constants of CO and $\mathrm{CO}_{2}$, we use $1.9225125 \mathrm{~cm}^{-1}$ and $0.39021894 \mathrm{~cm}^{-1}$ respectively. [25, 40]

We represent the Hamiltonian operator in a basis and compute eigenvalues. The basis functions used are

$$
f_{a_{0}}\left(r_{0}\right) u_{l_{1} l_{2} m_{2} K}^{J M P}\left(\theta_{1}, \theta_{2}, \phi_{2} ; \alpha, \beta, \gamma\right)
$$

where $f_{a_{0}}$ is a discrete variable representation (DVR) function [41] and $u_{l_{1} l_{2} m_{2} K}^{J M P}$ is a parity adapted rovibrational function[42, 43]. $\alpha, \beta, \gamma$ are Euler angles and $P=0,1$ correspond to even and odd 
parities. The DVR we use is a tri-diagonal Morse (TDM) DVR. [44] A TDM basis has three parameters: $D_{e}, \omega$, and $r_{e}$. The value of $D_{e}$ is the value obtained from the cut of the PES with $\theta_{1}$, $\theta_{2}$, and $\phi_{2}$ fixed at their values at the bottom of the C-in well. To determine $\omega$, and $r_{e}$, we choose values that give energy levels less than $140 \mathrm{~cm}^{-1}$ that are all within $.0001 \mathrm{~cm}^{-1}$ of the levels obtained with a large sine DVR basis. $\omega$ and $r_{e}$ are varied manually close to the values obtained from the cut used to choose $D_{e}$. The sine DVR basis is defined in the range (5.0 bohr, $38.0 \mathrm{bohr}$ ) and has 150 functions. The levels below $140 \mathrm{~cm}^{-1}$ above the zero point energy (ZPE) computed with the sine DVR basis have convergence errors smaller than $.0001 \mathrm{~cm}^{-1}$. The final $r_{0}$ basis has 20 tri-diagonal Morse DVR functions with the parameters $D_{e}=398 \mathrm{~cm}^{-1}, \omega=54.5 \mathrm{~cm}^{-1}$ and $r_{e}=6.85$ bohr. We use [44] $\alpha=A-2[A / 2]=0.606$, with $A=4 D_{e} / \omega_{e}$, in the associated Laguerre polynomial, so that all the bound states of the Morse Hamiltonian are exactly reproduced by the TDM basis. $u_{l_{1} l_{2} m_{2} K}^{J M P}$ are parity-adapted combinations of products of Wigner rotation functions, an associated Legendre function, and a spherical harmonic. They have amplitude everywhere in the angular configuration space and allow one to study large amplitude motion. For the angular basis, we used $l_{\max }=m_{\max }=45$. The $J=0 \mathrm{~A}+$ basis has about 329,000 functions.

Energy levels are calculated with the Lanczos algorithm [45-47] using the RV4 Code. [33] The full permutation-inversion (PI) group [48] is $G_{4}$. It has four irreducible representations: A+, $\mathrm{A}-, \mathrm{B}+$, and $\mathrm{B}-$, where $\mathrm{A} / \mathrm{B}$ label states that are symmetric/anti-symmetric under permutation of the two $\mathrm{O}$ atoms in $\mathrm{CO}_{2}$, and +/- label even/odd parity levels. The basis of Eq. (1) can be A/B symmetry-adapted by restricting $l_{2}$ to be even/odd, respectively. We therefore separately compute levels within each of the four $G_{4}$ symmetry blocks. To evaluate the matrix-vector products, sums are done sequentially.[41] Matrix elements of the kinetic energy operator are exact.[33, 35] Potential matrix elements are written as sums over quadrature points.[41, 49] We use $N_{\theta_{1}}=N_{\theta_{2}}=46$ Gauss-Legendre quadrature points and $N_{\phi_{2}}=92$ equally spaced trapezoid points in the range $[0,2 \pi]$. To reduce the spectral range of the Hamiltonian matrix (and accelerate Lanczos convergence) we use a potential ceiling [49] of $V_{\text {ceil }}=2098.4 \mathrm{~cm}^{-1}$.

\section{RESULTS}

Vibrational levels for both O-in and C-in isomers are listed in Table II with their respective quantum numbers as well as their symmetries. Energies are given with respect to the ZPE and, for states localised in the O-in well, we give in parenthesis energies with respect to the ZPE of 
TABLE II: Energy levels for both the C-in and O-in isomers. Energies in parenthesis are with respect to the O-in isomer's ground state energy. The number in front of the symmetry label is a cardinal number. A 2 before the quantum numbers indicates that the state is localized above the O-in well. $\mathrm{v}_{g}, \mathrm{v}_{a} \mathrm{v}_{t}, \mathrm{v}_{s}$ represent the geared, anti-geared, $\mathrm{CO}$ libration and stretch quantum numbers respectively. All energies are with respect to the ZPE, $-286.90 \mathrm{~cm}^{-1}$. The ZPE of the $\mathrm{O}$-in isomer is $111.383 \mathrm{~cm}^{-1}$ higher than the global minima, TC in Figure 1.

\begin{tabular}{|c|c|c|c|c|c|}
\hline $\mathrm{E}\left(E_{O-i n}\right)\left[\mathrm{cm}^{-1}\right]$ & n-sym & $\left(\mathrm{v}_{g}, \mathrm{v}_{a}, \mathrm{v}_{t}, \mathrm{v}_{s}\right)$ & $\mathrm{E}\left(E_{O-i n}\right)$ & n-sym & $\left(\mathrm{v}_{g}, \mathrm{v}_{a}, \mathrm{v}_{t}, \mathrm{v}_{s}\right)$ \\
\hline 0.0000 & $1 \mathrm{~A}+$ & $(0,0,0,0)$ & $94.1768(29.6795)$ & $8 \mathrm{~A}+$ & $2(2,0,0,0)$ \\
\hline 24.4546 & $1 \mathrm{~B}+$ & $(1,0,0,0)$ & 96.2892 & $9 \mathrm{~A}+$ & $(0,0,0,2)$ \\
\hline 44.5664 & 1B- & $(0,0,1,0)$ & $104.5450(40.0477)$ & $2 \mathrm{~A}-$ & $2(1,0,1,0)$ \\
\hline 47.3698 & $2 \mathrm{~A}+$ & $(2,0,0,0)$ & 104.9504 & $6 \mathrm{~B}+$ & $(5,0,0,0)$ \\
\hline 50.2140 & $3 \mathrm{~A}+$ & $(0,0,0,1)$ & $107.7748(43.2775)$ & $10 \mathrm{~A}+$ & $2(0,0,0,1)$ \\
\hline $64.4973(0.0000)$ & $4 \mathrm{~A}+$ & $2(0,0,0,0)$ & 107.9768 & $7 \mathrm{~B}+$ & \\
\hline 68.2473 & $1 \mathrm{~A}-$ & $(1,0,1,0)$ & 109.7627 & $3 \mathrm{~A}-$ & $(3,0,1,0)$ \\
\hline 68.8211 & $2 \mathrm{~B}+$ & $(3,0,0,0)$ & $110.1747(45.6774)$ & $8 \mathrm{~B}+$ & $2(3,0,0,0)$ \\
\hline 70.0601 & $3 \mathrm{~B}+$ & $(1,0,0,1)$ & 110.6554 & $9 \mathrm{~B}+$ & \\
\hline $79.1763(14.6790)$ & $4 \mathrm{~B}+$ & $2(1,0,0,0)$ & 111.7702 & $4 \mathrm{~A}-$ & \\
\hline 86.0042 & $5 \mathrm{~A}+$ & $(0,0,2,0)$ & 112.9367 & $10 \mathrm{~B}+$ & $(1,0,0,2)$ \\
\hline 87.9203 & $6 \mathrm{~A}+$ & $(4,0,0,0)$ & $113.3010(48.8039)$ & $11 \mathrm{~B}+$ & $2(0,1,0,0)$ \\
\hline $88.6163(24.12)$ & $2 \mathrm{~B}-$ & $2(0,0,1,0)$ & $114.0523(49.5550)$ & $11 \mathrm{~A}+$ & $2(0,0,2,0)$ \\
\hline 89.5665 & $5 \mathrm{~B}+$ & $(0,1,0,0)$ & 114.2612 & $12 \mathrm{~A}+$ & \\
\hline 90.2245 & $3 \mathrm{~B}-$ & $(2,0,1,0)$ & 119.8673 & $13 \mathrm{~A}+$ & \\
\hline 90.7751 & $7 \mathrm{~A}+$ & $(2,0,0,1)$ & $120.6303(56.1330)$ & $5 B-$ & \\
\hline 92.3644 & 4B- & $(0,0,1,1)$ & 123.3091 & $6 \mathrm{~B}-$ & $(0,0,3,0)$ \\
\hline
\end{tabular}

the lowest level localised in the O-in well. The energies in parenthesis can be directly compared with experimental transition frequencies for the O-in isomer. One important result is that the O-in ground state is $64.4973 \mathrm{~cm}^{-1}$ higher than the $\mathrm{C}$-in ground state, although the $\mathrm{O}$-in isomer well is $100.5 \mathrm{~cm}^{-1}$ higher than the $\mathrm{C}$-in isomer, as shown in Figure 1. This is due to the fact that the ZPE of the $\mathrm{C}$-in isomer is larger than the ZPE of the O-in isomer. Anti-geared vibrational states have 
higher energies than their geared counterparts $\left(24.4546\right.$ and $89.5665 \mathrm{~cm}^{-1}$ for the first geared and anti-geared states for the $\mathrm{C}$-in isomer respectively), because the wells are steeper in the anti-geared direction.

To make the vibrational assignments presented in Table II, we use 1-D and 2-D probability distribution (PD) and wavefunction plots. To make a 2-D (1-D) PD plot we integrate over the remaining 2 (3) coordinates. From the PD plots (not shown), it is clear that for the low-lying states, coupling between the stretch and other coordinates is weak. Our basic tool for making assignments is the nodal structure of wavefunction plots since they provide more information than PDs. PDs are less useful because a PD includes contributions from sums of squares of wavefunctions for all values of the other coordinates. For example, on the basis of the $\left(\theta_{1}, \theta_{2}\right)$ PD plot in Figure 7 a ( 8 a) for the geared (anti-geared) bending state at $24.4546 \mathrm{~cm}^{-1}\left(89.5665 \mathrm{~cm}^{-1}\right)$ one might conclude that $\theta_{1}$ and $\theta_{2}$ are weakly coupled (the contour lines are parallel to the axes), but wavefunction cuts in Figure $7 \mathrm{~b}$ (Figure $8 \mathrm{~b}$ ) reveal that at $\phi_{2}=0$ and $\phi_{2}=180^{\circ}$ the vibration occurs along the geared (anti-geared) coordinate. Figures $7 \mathrm{c}$ and $8 \mathrm{c}$ show that when $\phi_{2}=90^{\circ}$ the character of the vibrations changes so that in both cases vibration now occurs along $\theta_{2}$, this means that there is important coupling between $\phi_{2}$ and $\left(\theta_{1}, \theta_{2}\right)$.

To assign overtones and combinations, we use not only nodal structure of wavefunction plots, but also the energies of the fundamentals, e.g. once a fundamental is assigned, we look for its overtone near the energy that is about twice the energy of the fundamental. Finally, to confirm assignments we use also symmetry by using product rules for the group $\mathrm{C}_{2 v}$, e.g. if a state is labeled as $\mathrm{v}_{g}+\mathrm{v}_{t}$, its symmetry should be the product of both symmetries, $\mathrm{B}+$ and $\mathrm{B}-$, so this combination state should have an A- symmetry.

Figure $7 \mathrm{~b}$ and $\mathrm{c}$ (Figure $8 \mathrm{~b}$ and $\mathrm{c}$ ) show that although the $\phi_{2}=180^{\circ}$ wavefunction cut of the state we call the geared (anti-geared) fundamental has a node along the geared (anti-geared) coordinate, the $\phi_{2}=90^{\circ}$ wavefunction cut has nodes along $\theta_{2}$. A sequence of such geared states is shown in Figure 9. On the left, it is easy to recognize nodes along the geared coordinate; on the right, the same states have nodes along $\theta_{2}$. In Van der Waals dimers, geared states are very common (see for example Ref. 23), but this is the first time that geared states have been seen to change their character when $\phi_{2}$ is changed from $0^{\circ}$ to $180^{\circ}$.

Figure 5 shows PD plots for CO-libration states. They have nodes along $\theta_{1}$. The amplitude of the motion along $\phi_{2}$ is clearly large. The O-in isomer has a similar set of states. As a function of $\phi_{2}$, the PD is largest close to $\phi_{2}=90^{\circ}$. This CO bending therefore occurs when the two monomers 

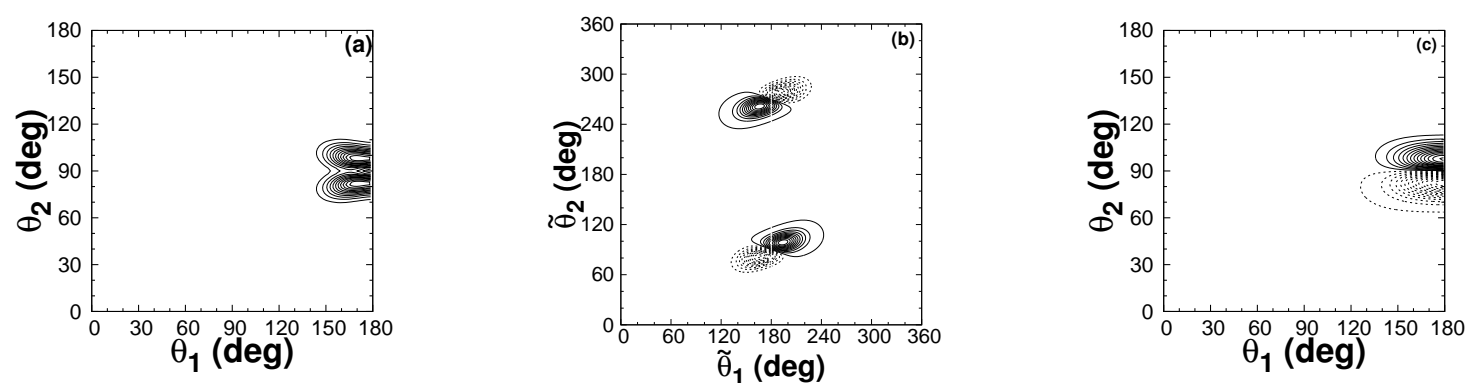

FIG. 7: Plots for the first geared state of the $\mathrm{C}$-in isomer at $24.4546 \mathrm{~cm}^{-1}(B+)$. a) Probability density as a function of $\theta_{1}$ and $\theta_{2} ; \mathrm{b}$ ) extended wavefunction cut as a function of $\theta_{1}$ and $\theta_{2} ;$ c) wavefunction cut as a function of $\theta_{1}$ and $\theta_{2}$ at $\phi_{2}=90^{\circ}$.
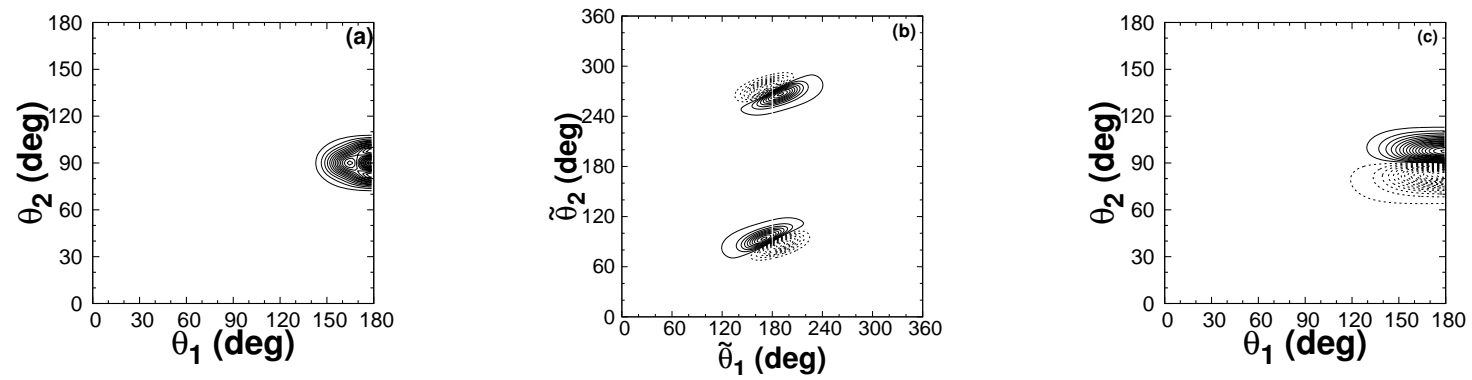

FIG. 8: Probability density plot (a), extended wavefunction cut (b) and wavefunction cut at $\phi_{2}=90^{\circ}$ (c) for the first anti-geared state of the C-in isomer, with energy of $89.5665 \mathrm{~cm}^{-1}(5 \mathrm{~B}+)$.

are in a cross configuration.

In Table III we compare the measured intermolecular frequencies we calculate with those obtained by Ford and coworkers.[6] The harmonic frequency for the B-vibration of the O-in isomer is the furthest from the experimental value. The fully coupled result is much closer. It is sometimes the case that higher wells are shallower and less harmonic. In previous papers $[2,3]$, the $\mathrm{B}+$ state is referred to as in-plane rock. In Table III, it is labelled as $\mathrm{v}_{g}$, where $g$ represents geared. Recall that wavefunction plots reveal that this state is geared when $\phi_{2}=180^{\circ}$ but a $\mathrm{CO}_{2}$ rock when $\phi_{2}=90^{\circ}$.

We have computed $J=1$ energy levels and they are reported, with assignments, in Tables IV and V. There are three closely spaced $J=1$ levels associated with each vibrational state. Knowing that the molecule is close to a prolate top, we assign the three levels assuming that $1_{01}<1_{11}<1_{10}$. Rotational constants are then obtained from the relations $1_{01}=\mathrm{B}+\mathrm{C}, 1_{11}=\mathrm{A}+\mathrm{C}$ and $1_{10}=\mathrm{A}+\mathrm{B}$. The rotational constants obtained from both isomer's ground states are compared to previous experimental and ab initio results in Tables VI and VII, and as it can be seen our constants 

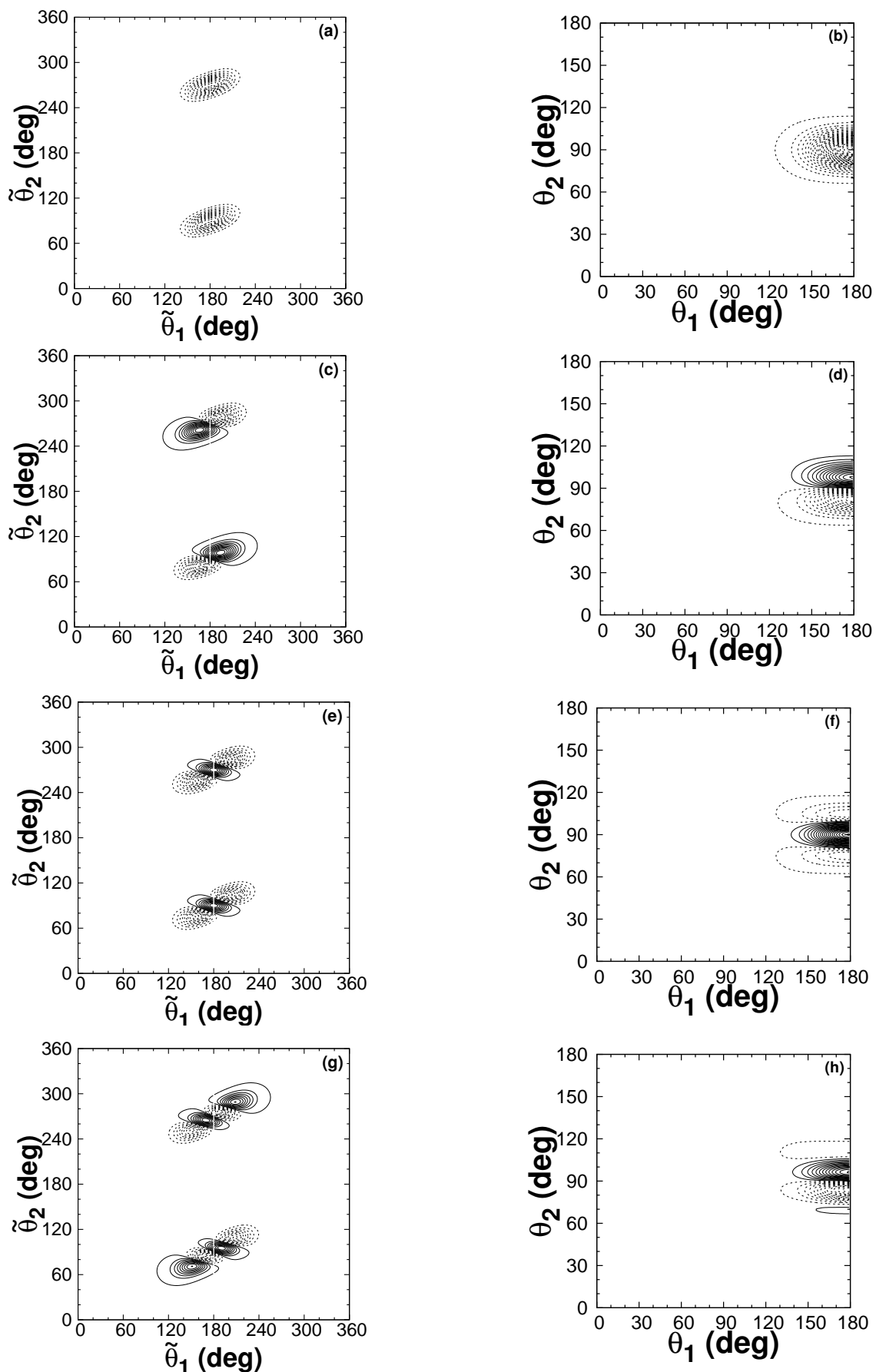

FIG. 9: Wavefunction cuts for the ground state [(a) and (b)] and the first 3 geared states [(c) and (d); (e) and (f); $(\mathrm{g})$ and $(\mathrm{h})]$ for the $\mathrm{C}$-in isomer whose energies are 0.0000(1A+), 24.4546(1B+), 47.3698(2A+) and $68.8211(2 \mathrm{~B}+) \mathrm{cm}^{-1}$. The wavefunction cuts on the left are in extended coordinates and show the in-plane behaviour of the molecule (both $\phi_{2}=0$ and $\phi_{2}=180^{\circ}$ ); the plots on the right are with $\phi_{2}=90^{\circ}$. All plots have a fixed value of $r=7.31 \mathrm{bohr}$, the equilibrium value for the $\mathrm{C}$-in isomer. The contour interval for the $\phi_{2}=90$ wavefunction cuts is about half the interval used in the extended plots, in order to show the $\theta_{2}$ nodal structure more clearly. 
TABLE III: Calculated and observed fundamental frequencies, in $\mathrm{cm}^{-1}$

\begin{tabular}{|c|c|c|c|c|c|c|c|}
\hline \multirow[b]{2}{*}{ Sym } & \multirow[b]{2}{*}{ State } & \multicolumn{3}{|l|}{$\mathrm{C}$-in isomer } & \multicolumn{3}{|l|}{ O-in isomer } \\
\hline & & Variational & Harmonic[6] & Observed [3] & Variational & Harmonic[6] & Observed [3] \\
\hline \multirow[t]{2}{*}{$\mathrm{B}+$} & $\mathrm{v}_{g}$ & 24.4546 & 24.30 & 24.343 & 14.6790 & 15.45 & 14.194 \\
\hline & $\mathrm{v}_{a}$ & 89.5665 & 90.79 & & 45.6774 & 51.62 & \\
\hline B- & $\mathrm{v}_{t}$ & 44.5664 & 42.81 & 43.958 & 24.1190 & 36.32 & 22.676 \\
\hline $\mathrm{A}+$ & $\mathrm{v}_{S}$ & 50.2140 & 56.39 & & 43.2775 & 55.37 & \\
\hline
\end{tabular}

TABLE IV: $\mathrm{J}=1$ rotational levels and rotational constants $\left(\mathrm{in}_{\mathrm{cm}}{ }^{-1}\right.$ ) of the $\mathrm{C}$-in isomer of $\mathrm{CO}-\mathrm{CO}_{2}$ for the fundamental vibrational states. Energies are relative to the ZPE of the $\mathrm{C}$-in isomer.

\begin{tabular}{lllllll}
\hline \hline $\mathrm{J}=0$ level & $1_{01}(\mathrm{sym})$ & $1_{11}(\mathrm{sym})$ & $1_{10}(\mathrm{sym})$ & $\mathrm{A}$ & $\mathrm{B}$ & $\mathrm{C}$ \\
$\left(\mathrm{v}_{g}, \mathrm{v}_{a}, \mathrm{v}_{t}, \mathrm{v}_{s}\right)$ & & & & & & \\
\hline $0.0000(0,0,0,0)(\mathrm{A}+)$ & $0.1165(\mathrm{~A}-)$ & $0.4495(\mathrm{~B}-)$ & $0.4584(\mathrm{~B}+)$ & 0.3957 & 0.0627 & 0.0538 \\
$24.4546(1,0,0,0)(\mathrm{B}+)$ & $24.5719(\mathrm{~B}-)$ & $24.9044(\mathrm{~A}-)$ & $24.9141(\mathrm{~A}+)$ & 0.3960 & 0.0635 & 0.0538 \\
$44.5663(0,0,1,0)(\mathrm{B}-)$ & $44.6838(\mathrm{~B}+)$ & $45.0166(\mathrm{~A}+)$ & $45.0250(\mathrm{~A}-)$ & 0.3957 & 0.0630 & 0.0545 \\
$50.2140(0,0,0,1)(\mathrm{A}+)$ & $50.3267(\mathrm{~A}-)$ & $50.6631(\mathrm{~B}-)$ & $50.6717(\mathrm{~B}+)$ & 0.3971 & 0.0606 & 0.0521 \\
$89.5665(0,1,0,0)(\mathrm{B}+)$ & $89.6798(\mathrm{~B}-)$ & $89.9900(\mathrm{~A}-)$ & $89.9984(\mathrm{~A}+)$ & 0.3711 & 0.0609 & 0.0525 \\
\hline \hline
\end{tabular}

agree well with previous experiments. The rotational constants obtained by assuming the molecule is rigid are much farther from the experimental values.

\section{CONCLUSION}

An accurate PES has been constructed and used to compute the low-lying energy levels of CO$\mathrm{CO}_{2}$. Energy levels were computed using a large spherical harmonic type basis and the Lanczos algorithm. Agreement with experimental band centers and rotation constants is excellent for both isomers. Using PD and wavefunction plots, energy spacing, and symmetry labels, it is possible to assign many states. We are able to confirm that the experimental frequency at $24.3 \mathrm{~cm}^{-1}$ is the geared fundamental. Referring to it as geared is, however, a bit of an oversimplification because although it is clearly geared when $\phi_{2}=0^{\circ}$, it is better described as a $\mathrm{CO}_{2}$ rock when $\phi_{2}=90^{\circ}$. Additionally, the CO libration was correctly identified and labelled for both isomers. 
TABLE V: $\mathrm{J}=1$ rotational levels and rotational constants $\left(\right.$ in $\mathrm{cm}^{-1}$ ) of the $\mathrm{O}$-in isomer of $\mathrm{CO}-\mathrm{CO}_{2}$ for the fundamental vibrational states. Energies are relative to the ZPE of the O-in isomer.

\begin{tabular}{lllllll}
\hline \hline $\mathrm{J}=0$ level & $1_{01}(\mathrm{sym})$ & $1_{11}(\mathrm{sym})$ & $1_{10}(\mathrm{sym})$ & $\mathrm{A}$ & $\mathrm{B}$ & $\mathrm{C}$ \\
$\left(\mathrm{v}_{g}, \mathrm{v}_{a}, \mathrm{v}_{t}, \mathrm{v}_{s}\right)$ & & & & & & \\
\hline $0.0000(0,0,0,0)(\mathrm{A}+)$ & $0.1374(\mathrm{~A}-)$ & $0.4593(\mathrm{~B}-)$ & $0.4719(\mathrm{~B}+)$ & 0.3969 & 0.0750 & 0.0624 \\
$14.6790(1,0,0,0)(\mathrm{B}+)$ & $14.8185(\mathrm{~B}-)$ & $15.1059(\mathrm{~A}-)$ & $15.1197(\mathrm{~A}+)$ & 0.3640 & 0.0767 & 0.0629 \\
$24.1190(0,0,1,0)(\mathrm{B}-)$ & $24.258(\mathrm{~B}+)$ & $24.6124(\mathrm{~A}+)$ & $24.6234(\mathrm{~A}-)$ & 0.4297 & 0.0753 & 0.0637 \\
$43.2775(0,0,0,1)(\mathrm{A}+)$ & $43.4130(\mathrm{~A}-)$ & $43.7998(\mathrm{~B}-)$ & $43.8117(\mathrm{~B}+)$ & 0.4605 & 0.0737 & 0.0618 \\
$48.8038(0,1,0,0)(\mathrm{B}+)$ & $48.9381(\mathrm{~B}-)$ & $49.2716(\mathrm{~A}-)$ & $49.2896(\mathrm{~A}+)$ & 0.4096 & 0.0761 & 0.0581 \\
\hline \hline
\end{tabular}

TABLE VI: Rotational constants for the ground, first geared, and CO-libration states of the C-in isomer. All values are in $\mathrm{cm}^{-1}$. Experimental values were obtained by Barclay et. al.[3]

\begin{tabular}{|c|c|c|c|c|c|c|c|}
\hline & \multicolumn{2}{|c|}{ Ground State } & \multicolumn{2}{|l|}{$\mathrm{v}_{g}$} & \multicolumn{2}{|l|}{$\mathrm{v}_{t}$} & \multirow[b]{2}{*}{ Ab initio Equib. 6 ] } \\
\hline & Variational & Exp. & Variational & Exp. & Variational & Exp. & \\
\hline $\mathrm{A}$ & 0.3957 & 0.3957 & 0.3960 & 0.3949 & 0.3957 & 0.3966 & 0.3850 \\
\hline $\mathrm{B}$ & 0.0627 & 0.0628 & 0.0635 & 0.0633 & 0.0630 & 0.0629 & 0.0608 \\
\hline $\mathrm{C}$ & 0.0538 & 0.0538 & 0.0538 & 0.0537 & 0.0545 & 0.0544 & 0.0525 \\
\hline
\end{tabular}

We have found three low-lying paths between the two isomers. They will influence the dynamics of higher states. One of the three paths is geared. It plays a role for many Van der Waals dimers. The second path is anti-geared. The third path is out-of-plane. Although TC is planar, any small change in $\theta_{1}$ puts the molecule into a region of the PES in which it is almost independent of $\phi_{2}$. Near $\phi_{2}=90^{\circ}$, it is feasible to change $\theta_{1}$ to convert the molecule from TC to TO. This may be

TABLE VII: Rotational constants for the ground, first geared and CO-libration states of the O-in isomer. All values are in $\mathrm{cm}^{-1}$. Experimental values were obtained by Sheybani-Deloui et. al.[2]

\begin{tabular}{|c|c|c|c|c|c|c|}
\hline \multicolumn{2}{|c|}{ Ground State } & \multicolumn{2}{|l|}{$\mathrm{v}_{g}$} & \multicolumn{2}{|l|}{$\mathrm{v}_{t}$} & \multirow[b]{2}{*}{ Ab initio Equib.[6] } \\
\hline Variational & Exp. & Variational & Exp. & Variational & Exp. & \\
\hline A 0.3969 & 0.3972 & 0.3640 & 0.3589 & 0.4297 & 0.4355 & 0.3850 \\
\hline B 0.0750 & 0.0745 & 0.0767 & 0.0762 & 0.0753 & 0.0749 & 0.0727 \\
\hline C 0.0624 & 0.0621 & 0.0629 & 0.0626 & 0.0637 & 0.0634 & 0.0612 \\
\hline
\end{tabular}


a common isomerization path between T-shaped isomers.

\section{ACKNOWLEDGEMENTS}

We are thankful for the support of the Canadian Natural Sciences and Engineering Research Council. Richard Dawes and Ernesto Quintas-SaÃn̆nchez are supported by the U.S. National Science Foundation (CHE-1566246).

[1] A. C. Legon and A. P. Suckley, The Journal of Chemical Physics 91, 4440 (1989).

[2] S. Sheybani-Deloui, A. J. Barclay, K. H. Michaelian, A. R. W. McKellar, and N. Moazzen-Ahmadi, Journal of Chemical Physics (2015).

[3] A. J. Barclay, S. Sheybani-Deloui, K. H. Michaelian, A. R. McKellar, and N. Moazzen-Ahmadi, Chemical Physics Letters 651, 62 (2016).

[4] R. W. Randall, J. P. L. Summersgill, and B. J. Howard, J. Chem. Soc., Faraday Trans. 86, 1943 (1990).

[5] V. Kellö, K. P. Lawley, and G. H. Diercksen, Chemical Physics Letters 319, 231 (2000).

[6] M. Venayagamoorthy and T. A. Ford, in Journal of Molecular Structure (2001).

[7] K. M. de Lange and J. R. Lane, The Journal of Chemical Physics 134, 034301 (2011).

[8] D. J. Nesbitt, Chemical Reviews (1988).

[9] Z. Bačić and R. E. Miller, Journal of Physical Chemistry (1996).

[10] D. H. Zhang, Q. Wu, J. Z. Zhang, M. Von Dirke, and Z. Bačić, The Journal of Chemical Physics (1995).

[11] M. Dehghany, M. Rezaei, N. Moazzen-Ahmadi, A. McKellar, J. Brown, X.-G. Wang, and T. Carrington, Journal of Molecular Spectroscopy 330, 188 (2016).

[12] X.-G. Wang and T. Carrington, The Journal of Chemical Physics 143, 024303 (2015).

[13] H. Cybulski, C. Henriksen, R. Dawes, X.-G. Wang, N. Bora, G. Avila, T. Carrington, and B. Fernández, Physical Chemistry Chemical Physics 20, 12624 (2018).

[14] A. J. Barclay, A. R. W. McKellar, N. Moazzen-Ahmadi, R. Dawes, X.-G. Wang, and T. Carrington, Physical Chemistry Chemical Physics 20, 14431 (2018).

[15] R. Dawes, X.-G. Wang, and T. Carrington, The Journal of Physical Chemistry A 117, 7612 (2013). 
[16] G. W. M. Vissers, A. Hebelmann, G. Jansen, P. E. S. Wormer, and A. van der Avoird, The Journal of Chemical Physics 122, 54306 (2005).

[17] L. A. Surin, D. N. Fourzikov, T. F. Giesen, S. Schlemmer, G. Winnewisser, V. A. Panfilov, B. S. Dumesh, G. W. M. Vissers, and A. van der Avoird, The Journal of Physical Chemistry A 111, 12238 (2007).

[18] H. Li, P.-N. Roy, and R. J. Le Roy, The Journal of Chemical Physics 132, 214309 (2010).

[19] G. Guelachvili, Journal of Molecular Spectroscopy 79, 72 (1980).

[20] H.-J. Werner, P. J. Knowles, G. Knizia, F. R. Manby, and M. Schütz, Wiley Interdisciplinary Reviews: Computational Molecular Science 2, 242 (2012).

[21] H.-J. Werner, G. Knizia, and F. R. Manby, Molecular Physics 109, 407 (2011).

[22] K. A. Peterson, T. B. Adler, and H.-J. Werner, The Journal of Chemical Physics 128, 84102 (2008).

[23] G. Donoghue, X. G. Wang, R. Dawes, and T. Carrington, Journal of Molecular Spectroscopy 330, 170 (2016).

[24] X.-G. Wang, T. Carrington, and R. Dawes, Journal of Molecular Spectroscopy 330, 179 (2016).

[25] J. Brown, X.-G. Wang, T. Carrington, G. S. Grubbs, and R. Dawes, The Journal of Chemical Physics 140, 114303 (2014).

[26] R. Dawes and E. Quintas-Sánchez, in Reviews in Computational Chemistry vol. 31 (2018), chap. 5, pp. 199-264.

[27] E. Quintas-Sánchez and R. Dawes, Journal of Chemical Information and Modeling 59, 262 (2019).

[28] J. Brown, X. G. Wang, R. Dawes, and T. Carrington, Journal of Chemical Physics (2012).

[29] X.-G. Wang, T. Carrington, R. Dawes, and A. W. Jasper, Journal of Molecular Spectroscopy 268, 53 (2011).

[30] V. Babin, C. Leforestier, and F. Paesani, Journal of Chemical Theory and Computation (2013).

[31] C. Leforestier, L. B. Braly, K. Liu, M. J. Elrod, and R. J. Saykally, Journal of Chemical Physics (1997).

[32] C. Leforestier, The Journal of Chemical Physics (1991).

[33] R. Dawes, X. G. Wang, A. W. Jasper, and T. Carrington, Journal of Chemical Physics (2010).

[34] P. Sarkar, N. Poulin, and T. Carrington, The Journal of Chemical Physics 110, 10269 (1999).

[35] X.-G. Wang, T. Carrington, J. Tang, and A. R. W. McKellar, The Journal of Chemical Physics 123, 034301 (2005).

[36] S. Y. Lin and H. Guo, Journal of Chemical Physics (2003).

[37] H. G. Yu, H. Song, and M. Yang, Journal of Chemical Physics (2017). 
[38] G. Brocks, A. van der Avoird, B. Sutcliffe, and J. Tennyson, Molecular Physics 50, 1025 (1983).

[39] X. Chapuisat and C. Iung, Physical Review A 45, 6217 (1992).

[40] R. a. Toth, Journal of the Optical Society of America B (1987).

[41] J. C. Light and T. Carrington, Advances in Chemical Physics 114, 263 (2000).

[42] X.-G. Wang and T. Carrington, The Journal of Chemical Physics 121, 2937 (2004).

[43] X.-G. Wang and T. Carrington, The Journal of Chemical Physics 118, 6946 (2003).

[44] H. Wei and T. Carrington, The Journal of Chemical Physics 97, 3029 (1992).

[45] X.-G. Wang and T. Carrington, The Journal of Chemical Physics 115, 9781 (2001).

[46] X.-G. Wang and T. Carrington, The Journal of Chemical Physics 114, 1473 (2001).

[47] R. Chen and H. Guo, The Journal of Chemical Physics 114, 1467 (2001).

[48] P. R. Bunker, P. Jensen, and National Research Council Canada., Molecular symmetry and spectroscopy (NRC Research Press, 1998).

[49] M. J. Bramley, J. W. Tromp, T. Carrington, and G. C. Corey, The Journal of Chemical Physics 100, 6175 (1994). 